\title{
Study on Synthetic Reinforcing Approach and Its Applications
}

\author{
Bing XING ${ }^{1, a^{*}}$, Jun XIAO ${ }^{2, b}$, Shi-zhong QIANG ${ }^{3, c}$,Tang TANG ${ }^{4, d}$ \\ ${ }^{1}$ School of Civil Eng., Southwest Jiaotong University, Chengdu 610031, China;\&Sichuan Huateng \\ road test for detection of limited liability company, Chengdu 611730, China \\ ${ }^{2}$ School of Civil Eng., Southwest Jiaotong University, Chengdu 610031, China \\ ${ }^{3}$ School of Civil Eng., Southwest Jiaotong University, Chengdu 610031, China \\ ${ }^{4}$ School of Civil Eng., Southwest Jiaotong University, Chengdu 610031, China \\ a290827045@qq.com, bsunflower001@foxmail.com, \\ cqiangshizhong@163.com, ${ }^{\mathrm{d}} 447248080 @ q q . c o m$
}

\begin{abstract}
Keywords: Structure prestress; Synthetic reinforcing approach; Adjustment of internal force; Additional forces of temperature difference; Application examples

Abstract: A new method of synthetic reinforcing approach is first put forward systematicly in this paper. Its main feature is the old and new material obtainted the appropriate prestressing by adjusting structural reinforcement of internal forces, realizing the combination of the structure prestress technology and the traditional increasing section method, which can effectively solve the new material stress reinforcement hysteresis and strength problem. Firstly, the concept of the structure, the key points and engineering measures of the comprehensive strengthening method are introduced, and the effect of the method is verified by engineering examples.
\end{abstract}

\section{Introduction}

Active reinforcement method and passive reinforcement method are two main ways of reinforcing at present Active reinforcement includes external prestressing reinforcement,bonded prestressed reinforcement, and CFRP method, while passive reinforcement method includes section increasing , steel plate pasting and carbon fiber material method.Recent years,two kinds of trends are presented in the field of bridge reinforcement. One is changing passive to active and fully exert the high tensile strength of the post reinforcing material. Effectively improve the utilization of its materials. Overcome the strain (stress) reinforcement hysteresis of the post reinforcing material based on prestressing reinforcement method. The other is changing single reinforcement to comprehensive reinforcement. As any reinforcement method has certain advantages and disadvantages and limitations, its scope of application is restricted by the reason of bridge reinforcement, the characteristics of structure, environment and economy. Sinceit's hard to find a way to adapt to all the problems, many researchers have proposed a comprehensive reinforcement method which contains a variety of single reinforcement methods. In order to effectively solve the defects of single rand passive reinforcement method and apply effective prestress to the strengthened members, composite reinforcement method of prestressed structure is put forward for the first time in this paper.Taking a bridge strengthening and maintenance engineering as an example, the paper introduces the design, process and engineering measures of the composite strengthening method, which is verified by the engineering practice.

\section{Introduction to the structural prestressed reinforcement}

\section{The concept of structure prestress}

Inspired by the construction technology of the pre bending beam and considering that the pre bending beam can obtain the ideal prestress without any tension equipment and prestressed wire, the author defines structure prestress as follows:structure prestress: Firstly, adjust the internal force of the original structure temporarily and use passive reinforcement method to reinforce the original structure 
to ensure the effective bonding of the new and old structure layer .Then,release temporary internal force and make both the old and new reinforced materials obtain the prestressing force. The stress essence of structure prestress is consistent with traditional pre bending prestress that is the active deformation of the structure is the source of the prestressing force. Moreover, the type and manner of the prestress application is expanded on the base of the pre bending beam, providing more solutions for bridge reinforcement. 'Pre bending' is the only way for pre bending beam to obtain prestress. That is by changing the moment of a particular section of the original structure. However, for bridge member that needed to be reinforced, the internal force of corresponding control section that need to be changed may be bending moment, axial force and shear force, or two or three of them. Therefore, we should apply the corresponding prestressing force to the structure according to the requirement in reinforcing design. Not only taking 'pre bonding' into account, but also considering 'pre compression', 'pre tension', 'precut', 'pre bending and tension', 'pre bending and compression', 'pre bending and cut', etc.Thereby obtaining prestressing force which is needed or useful. For different types of bridges, we can choose different types and manners of structure prestress application according to the characteristics of the concrete structure system, the weak links and other factors. Which kind of prestress to take depends on the status of the bearing capacity and the requirements of the designer.

\section{Structure prestressed reinforcement method}

The comprehensive reinforcing method is a method which combines the structure prestress technology and the traditional method of increasing the cross section (including the other materials such as the steel plate or carbon fiber). This method is a kind of comprehensive reinforcing method, which is used to combine the passive reinforcement method and the active reinforcement method, and by temporarily adjustment of internal force, so as to obtain the effective pre tension (or compression) stress.

By means of an early structure (or cross section), the deformation generated by the internal force adjustment can be generated by the loading of the material (or the cross section) of the loading process. The deformation of the early structure (or cross section) generated by the internal force adjustment can be restricted. The acquisition of the prestressing force of the structure is realized by adjusting the internal force of the original structure. Its 'internal force adjustment' mainly refers to the 'temporary internal force adjustment'. That is to be a temporary internal force adjustment, the conventional passive reinforcement, waiting for the old and new materials, the new material strength to meet the requirements of the temporary adjustment of internal forces, so that the new material (or old materials) to obtain the desired prestress. This temporary internal force adjustment does not change the internal force of the cross section of the reinforcement, but the distribution of the stress in the cross section is changed (that is, the pre stress) is obtained. The method not only includes the temporary internal force adjustment measures, but also uses the traditional 'unloading reinforcement method' to unload the deck dead load, followed by passive method reinforcing the main structure and reconstruct bridge deck sections ideas to achieve. It is an indirect reinforcement method by reducing the thickness of the bridge deck pavement or replacing the unloading and strengthening of the lightweight material and not belongs to the structural prestressed reinforcement method. Structure prestressed reinforcement method combines the traditional passive reinforcement method with the structure of prestressed technology and has the following advantages:

(1) It is effective to control the stress difference between the old and the new materials, so that the old and new materials can give full play to the strength of the material.

(2) Eliminates complex construction tensioning and anchoring and don't need any corresponding equipment.

(3) It can greatly improve the effect of the indirect reinforcement method which is commonly used and mature.

(4) The mode of prestress application is varied according to different structures, which can solve the construction conditions of the reinforcement method to restrict the problem. 
(5) For the bridge with a large proportion of dead load, it's very difficult for the conventional method of active reinforcement to carry out effectively, but this method provides an effective and feasible solution.

(6) The way of prestress application is simple. The structure stress is clear. This greatly reduces the level requirements for people engaged in bridge reinforcement design and construction and makes the quality of the reinforcement easy to be guaranteed.

(7) This method greatly improves the economic benefit and social benefit of bridge reinforcement.

\section{Engineering Measures for Structure prestressed reinforcement method}

As a kind of active reinforcement method, structure prestressed reinforcement method is suitable for various bridge structures. This section mainly takes the arch bridge and the small and medium span simply supported beam as an example and studies method of its internal force adjustment, i.e., studying the main engineering measures for prestressed application.

\section{Structure prestressed reinforcement method for arch bridge}

\section{Internal force adjustment of the bridge deck unloading}

Unloading on deck is a direct and convenient method for internal force adjustment. The traditional method of unloading is based on the principle of influence line. Its internal force adjustment measures are limited by the need to replace the bridge deck. The internal force adjustment is directly related to the weight of the bridge deck system. And unloading on the different positions of the bridge deck have different influences on the control section of the main arch. Therefore, to reinforce specific control section of hingeless arch, we should select appropriate reinforcement method. When reinforcing the arch foot section A, crown section B and arch foot section C, we can choose to remove the bridge deck system in "positive (negative) moment area". As shown in Fig 1, generally, unloading at a certain position of the bridge deck system is hard to meet the needs of multiple control section reinforcement of the main arch at the same time. Therefore, we need to consider the order of reinforcement and the combination of different unloading methods.

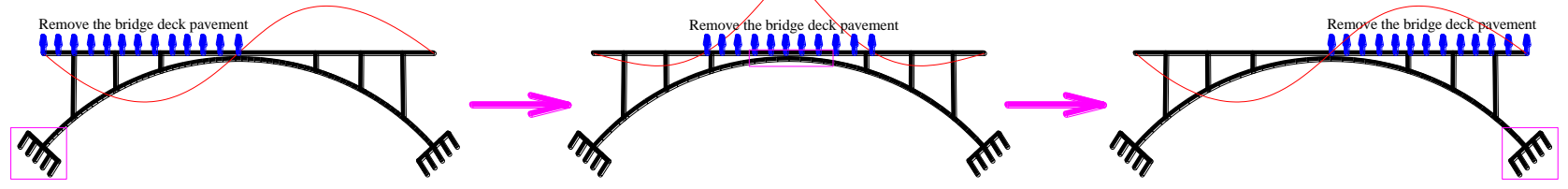

Fig.1 Adjustment of the internal force of the bridge deck

\section{Internal force adjustment method of bridge deck loading}

For arch bridge of which the deck system cannot be replaced or if the weight of the bridge deck system is insufficient to meet the design requirements of the unloading amount of reinforcement, we can take deck temporary loading mode to achieve internal force adjustment. This method is based on the principle of influence line. As shown in Fig 2, for removing dead load negative moment, we can load at the positive moment region of the influence line. Contrarily, if removing dead load positive moment, choose to load at the negative moment region of the influence line. Under the premise of ensuring the safety of bridge structure, temporary load of the bridge deck should be controlled according to the most adverse effect of bridge's live load. This method can effectively utilize the bearing capacity of the bridge. 


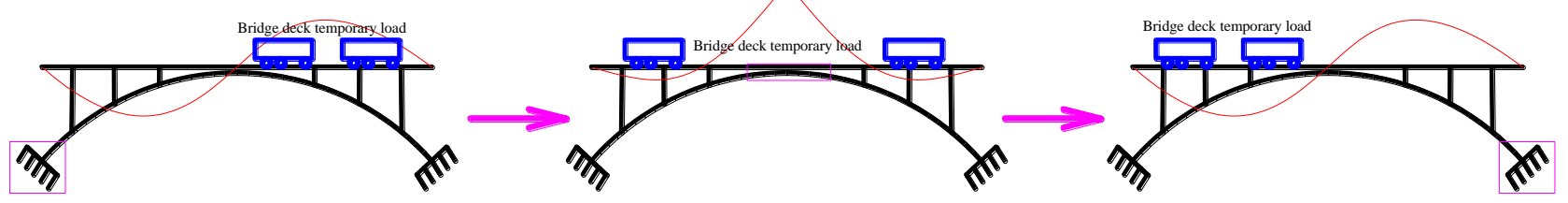

Fig.2 Bridge deck loading internal force adjustment method

\section{Environmental temperature difference internal force adjustment method}

Temperature difference can lead to the adjustment of the structural internal force of the non-hinged arch bridge. Therefore, when reinforcing control section of a non-hinged arch bridge, we can select a specific temperature field for strengthening the construction to achieve the reinforcement effect. When using increasing section method to reinforce the arch foot section of which the negative moment is large, we can select to reinforce in the high temperature in summer. The temperature effect of the arch foot section has a large positive moment, which makes the negative moment of the cross section is smaller. When the bridge deck system works well and long time traffic control has a certain degree of difficulty, it's better to choose to reinforce on the main archat noon in summer. Similarly, when positive moment existing inarch foot section and negative moment existing in arch crown section, it's better to choose to reinforce on the main arch at noon in summer. The existence of negative bending moment in the section of arch foot is positive moment and arch crown at winter evening.

\section{Prestressed composite reinforcement method for bridge structure}

\section{Lift the mid-span}

Most of the small and medium span girder bridges are simple supported structures. Disease often occurs at the cross section because of the maximum positive bending moment. For the part of the beam that is not easy to build up the bracket through the bottom of the beam, the beam will be temporarily shared to the adjacent beams. Based on this, a cross reference is proposed to unload. The main steps are as follows: (1)Make the diaphragm, the roof joint, deck pavement of the T beam bridge cut along the longitudinal direction of the bridge. (2) Drill in the roof and use thread steel to connect the $\mathrm{T}$ beam to be reinforced and the distribution beam. (3)Plant reinforcing bar, chisel, stand formwork in a cross section that needs to be reinforced. (4)Arrange the jack in the anti-forced beam and control the jack, reach a predetermined tonnage. (5)Pour reinforcing layer concrete, cure concrete until meeting strength requirements. (6)Remove jack on the top step by step. (7)Repour the diaphragm.
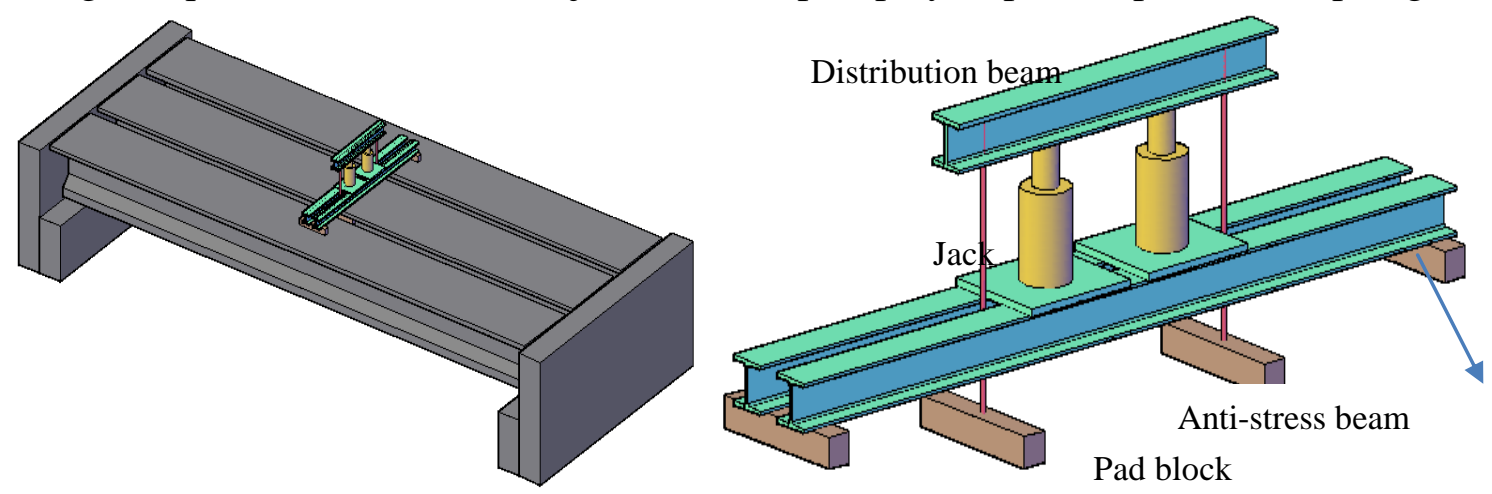

Fig.3 Sketch map of lifting the mid-span

\section{Temporary external prestressing}

According to the principle of prestressed concrete beam bridge, the method of applying temporary external prestress to the lower edge of the $\mathrm{T}$ beam can efectively reduce the positive bending moment. 
To reduce the tonnage of the jack, using pulley block, we develop a set of equipment for tension, as shown in Fig4. (1)Make the diaphragm, the roof joint, deck pavement of the $\mathrm{T}$ beam bridge cut along the longitudinal direction of the bridge. (2)Plant reinforcing bar, chisel, stand formwork in a cross section that needs to be reinforced. (3)Install the anti force plate and the tension equipment at the beam end. (4)Control the hoister, reach a predetermined tonnage. (5)Pour reinforcing layer concrete, cure concrete until meeting strength requirements. (6)Release the hoist. (7)Repour the diaphragm.
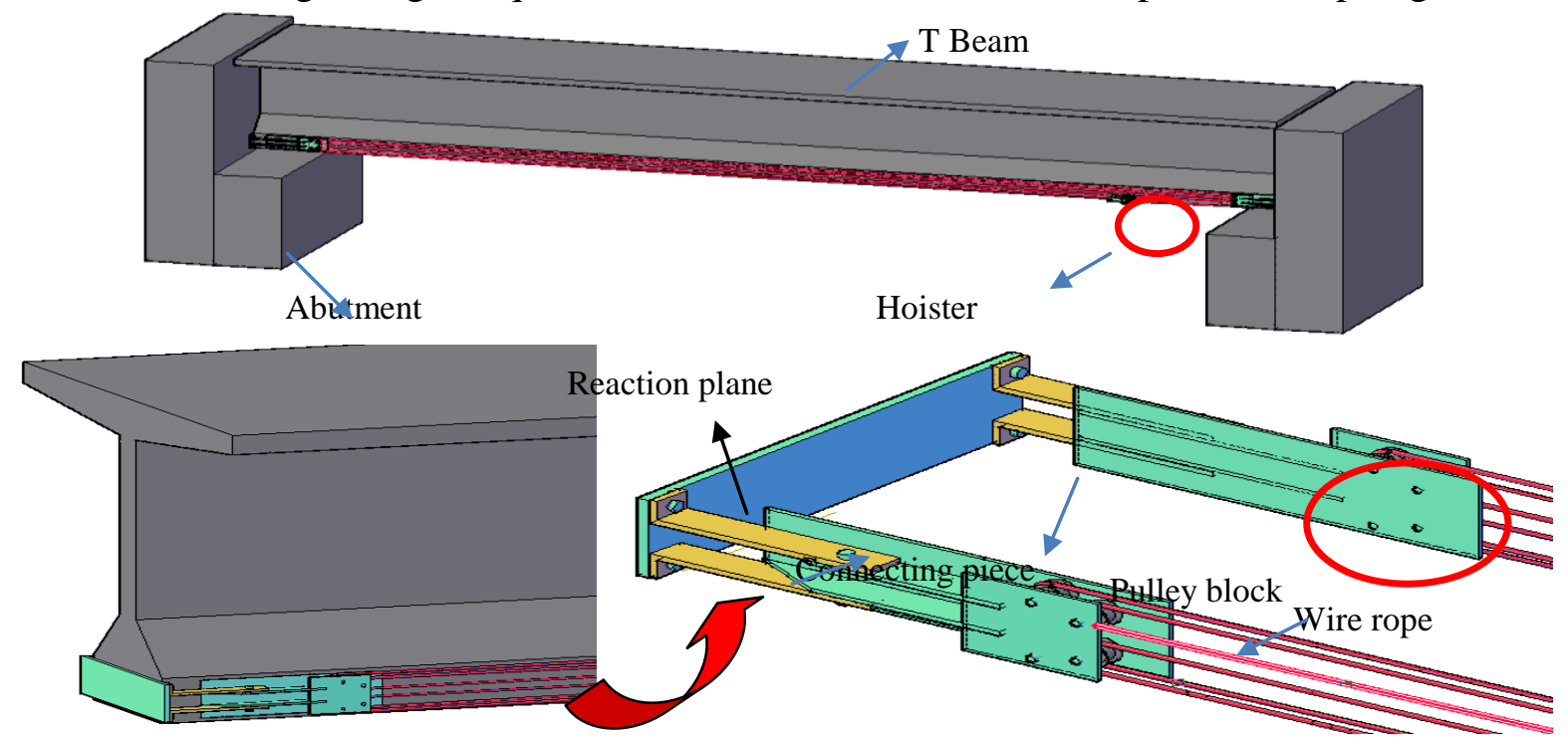

Fig.4 Sketch map of temporary external prestressing

\section{Temporary loading on the top beam}

For simply supported $\mathrm{T}$ beam bridge whose two ends are abutments, we can easily make the the front wall of abutment as "reaction wall".Therefore, we put forward the following unloading measures. Specific construction process is as follows: (1)Make the diaphragm, the roof joint, deck pavement of the $\mathrm{T}$ beam bridge cut along the longitudinal direction of the bridge. The side of the horseshoe is anchored in the side. (2)Plant reinforcing bar, chisel, stand formwork in a cross section that needs to be reinforced. (3)Arrange the jack at the bottom of the beam, Control the jack, reach a predetermined tonnage. (4) Pour reinforcing layer concrete, cure concrete until meeting strength requirements. (5)Remove jack on the top step by step. (6) Repour the diaphragm.

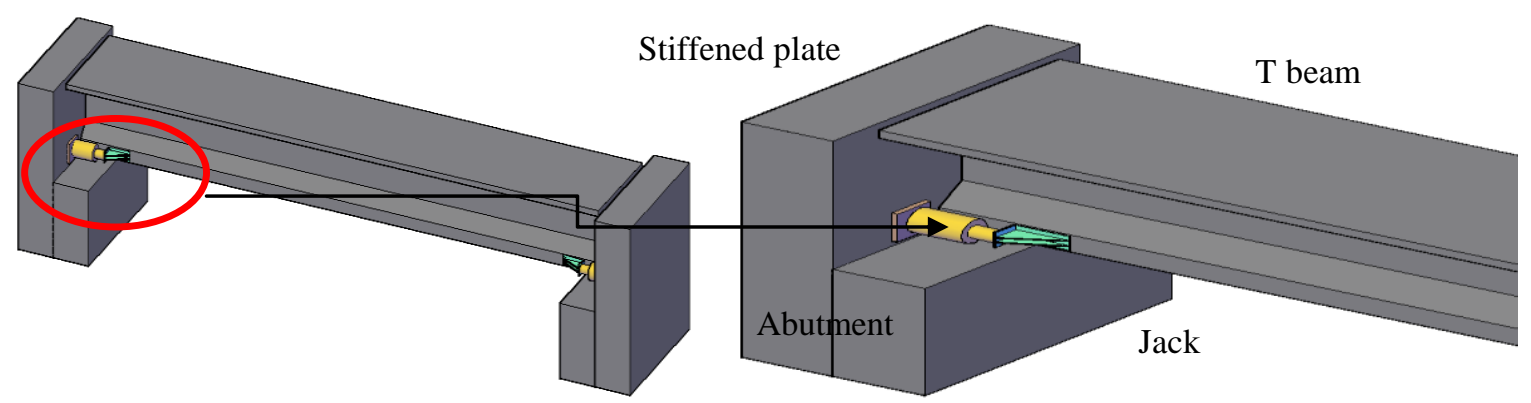

Fig.5 Sketch map of temporary loading method 


\section{Engineering application}

\section{Engineering background}

The length of the bridge is $339.72 \mathrm{~m}$, the main span is a fasting type of box plate which is $3 * 100 \mathrm{~m}$. Design load: twenty cars, trailer load -100 . The bridge is reinforced with the structural prestress, and the reinforcement is shown in Fig 3.

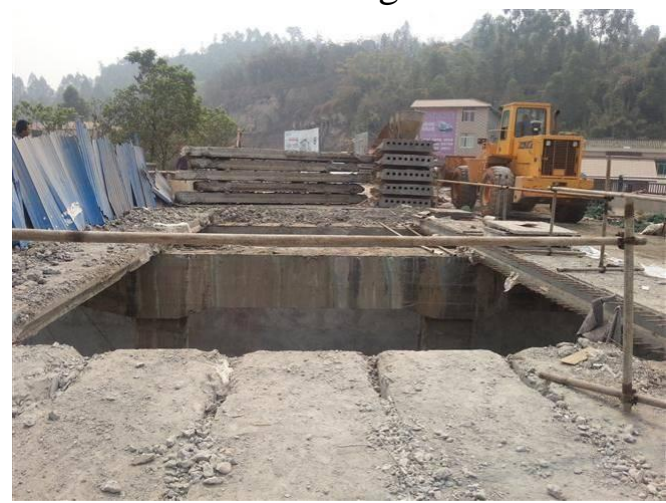

(a) Remove the deck

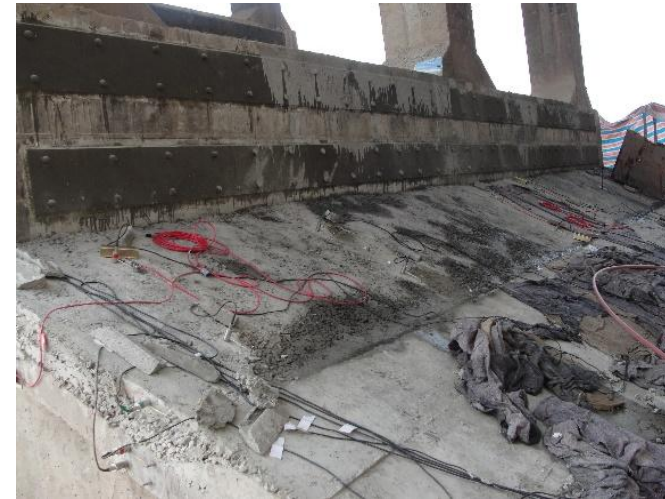

(b) Arch foot reinforcement

Fig.6 Application examples of structural prestress

This design uses the comprehensive reinforcement method, mainly for the reinforcement of arch foot section of the main arch circle First of all, remove the bridge deck which increases the positive moment of arch foot section and then, use the enlarging section area method to enlarge the arch foot section for reinforcement. Finally, when the reinforcing new materials strength meet the design requirements, remove the temporarily internal force adjustment measures. This method is equal to increase the positive moment to the reinforcement section (arch foot section), after the end of this reinforcement process, the concrete of the new section will get some of the stress reserve, and the stress reserve of the concrete at the old section will be reduced, in this way, we can make a result that can reduce the stress difference between the concrete of the old section and the concrete of the new section. The reinforcement flow chart of the bridge structure is shown in Fig 7.

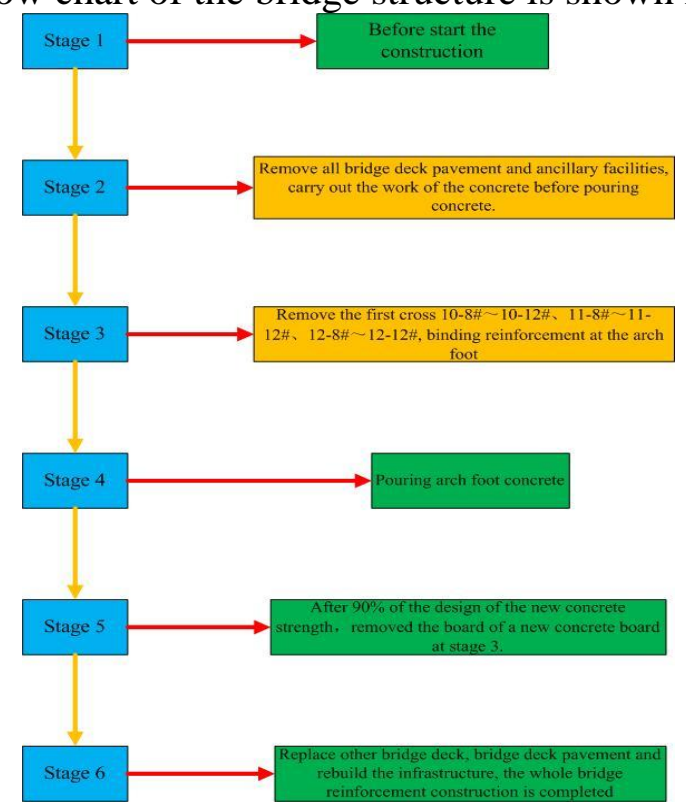

Fig.7 Flow chart of comprehensive reinforcing of bridge structure

\section{Effect evaluation of structural prestressed reinforcement}

By evaluating the increase of the cross section strengthening method and the structure of the prestressed reinforcement method in the old and new materials to determine the level of stress level of prestressed reinforcement method. 


\section{New and old section stress state after reinforcement}

The stress comparison chart of old and new concrete before and after the reinforcement at the outer edge of the bridge arch foot section is shown in Fig 8. From the picture, after comprehensive reinforcement of structure, the stress difference between the new and old concrete at the arch foot section is reduced, the stress lag of the new and old materials has been relieved.

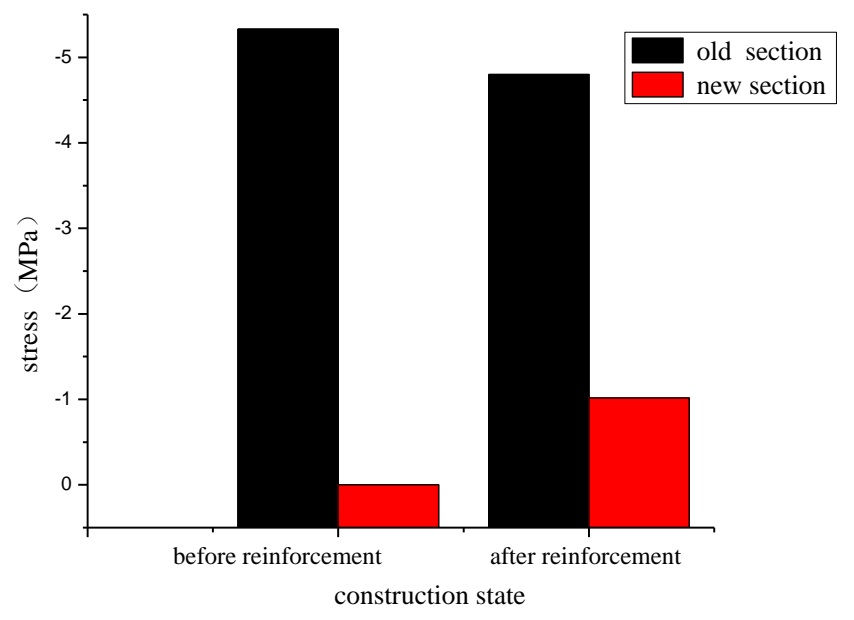

Fig.8 Stress contrast of new and old concrete at the arch foot section

\section{The contrast between Increasing section and prestressed reinforcement method}

In order to quantitatively describe the effect of the reinforcement on the bridge. First, do not consider the case of unloading and calculate the stress state of the new and old materials which is only reinforced by increasing the section method. Then, comparing with the method of increasing section method. The stress contrast data is shown in Table 1.

\begin{tabular}{|c|c|c|c|c|c|}
\hline \multicolumn{3}{|c|}{ Position } & \multicolumn{2}{|c|}{$\begin{array}{l}\text { Stress state of constant load after } \\
\text { reinforcement }(\mathrm{MPa})\end{array}$} & \multirow{2}{*}{$\begin{array}{c}\text { Differen } \\
\text { ce } \\
(\mathrm{MPa})\end{array}$} \\
\hline Section & Description & $\begin{array}{l}\text { Distance } \\
\text { to bottom } \\
\quad(\mathrm{m})\end{array}$ & $\begin{array}{l}\text { Increasing } \\
\text { section }\end{array}$ & $\begin{array}{c}\text { Structural } \\
\text { prestressed } \\
\text { reinforcement }\end{array}$ & \\
\hline \multirow{4}{*}{$\begin{array}{l}\text { Arch foot } \\
\text { section }\end{array}$} & $\begin{array}{c}\text { upper } \\
\text { border of } \\
\text { new } \\
\text { material }\end{array}$ & 1.85 & 0.00 & -1.02 & -1.02 \\
\hline & $\begin{array}{c}\text { Lower } \\
\text { border of } \\
\text { new } \\
\text { material }\end{array}$ & 1.74 & 0.00 & -1.05 & -1.05 \\
\hline & $\begin{array}{c}\text { Upper } \\
\text { border of } \\
\text { old material }\end{array}$ & 1.70 & -5.40 & -4.86 & 0.54 \\
\hline & $\begin{array}{c}\text { Upper } \\
\text { border of } \\
\text { old material }\end{array}$ & 0.00 & -10.17 & -10.19 & -0.02 \\
\hline
\end{tabular}

Table 1 Two kinds of methods for reinforcing the old and new materials

(Note: The positive value indicates tensile stress and the negative indicates pressure stress.)

Comparing this two kinds of reinforcement methods, we can find that the new material is non stress state after traditional reinforcing method. When using this new method to reinforce the 
structure, the new material of the arch foot section is in a state of compression, the upper and lower values are 1.05MPa and 1.02MPa. Compared with reinforcing the cross section of the arch foot by increasing the section method, the comprehensive reinforcing method is equivalent to the storage of prestress in the cross section of the arch foot and has achieved a good reinforcement effect.

\section{Conclusion}

This paper puts forward the concept of the structure, the main points of construction design for the first time. On the basis of the above, the paper puts forward the comprehensive reinforcing method of structural stress. The mechanism of structural stress and the characteristics of the new and old materials of the new and old materials in bridge strengthening are highly combined. So as to provide an effective solution for the research of bridge reinforcement, the 'stress lag', the strength of the new material can not be fully played in bridge reinforcement. And combined with the practice of a bridge reinforcement project, the method is proved to be convenient and easy to operate, and have achieved ideal reinforcement effect.

\section{References}

[1]ZHOU Yong-tao,BAO Wei-gang,LIU Yan-fang, et al.Study of Key Techniques for Bridge Strengthening Engineering [J]. Bridge Construction. 2010(6): 49-52.

[2]YU Tian-qing,JIANG Yong-hong. Research and Application of Arch Bridge Strengthening Techniques [J]. World Bridges. 2006(1): 74-76.

[3] GUO Da-jin,LIAO Jin-xiang,ZHANG Jing-quan,et al. Application of Bridge Strengthening Technology [J]. Journal of Highway and Transportation Research and Development. 2004, 21(8): 71-73.

[4]Du Yangyang. Research on new technology of highway bridge reinforcement[J]. Transpo World. 2013(9): 200-201.

[5]Zhou Y, Gou M, Zhang F, et al. Reinforced concrete beams strengthened with carbon fiber reinforced polymer by friction hybrid bond technique: Experimental investigation[J]. Materials \& Design. 2013, 50: 130-139.

[6]BIAN Jin-mei,ZHU Fu-shen,GAO Xiao-gang, et a. Optimization decision support system for bridge reinforcement scheme [J]. Journal of China \& Foreign Highway. 2009, 29(2): 156-161.

[7] Sun Baojun,Li Bingnan,et al.Integrated strengthening technique of concrete structures and its applications [J]. Industrial Architecture. 2003(05): 74-77.

[8] Zhang Shiduo. Introduction of pre-bending prestressed concrete beams [J]. Highway. 1987(03): $2-5$.

[9] Yin Dingan,Zhang Shiduo. A new type of Prestressed Structure -- pre bending prestressed concrete highway bridge [Z]. Wuhan, Hubei, china: 661-666.

[10] Zhou Zhixiang,Feng Zhiyong,Wang Zhenyin. PFRC Beam and Their Application in Tongxing Bridge [J]. Journal of Chongqing Jiaotong Institute. 1996, 15(Suppl.): 8-13. 